\title{
LA PLANIFICACIÓN ESTRATÉGICA Y LA CALIDAD DE LA EDUCACIÓN EN LAS INSTITUCIONES EDUCATIVAS DE NIVEL BÁSICO ELEMENTAL.
}

\section{STRATEGIC PLANNING AND THE QUALITY OF EDUCATION IN THE ELEMENTARY BASIC EDUCATIONAL INSTITUTIONS.}

\author{
Autor: Marcos Oswaldo García Romero \\ E-mail contacto: marcososwaldo2007@ hotmail.com \\ ORCID ID: https://orcid.org/0000-0002-7683-4576 \\ Articulo recibido: 16 de Mayo del 2019 \\ Articulo revisado: 7 de Julio del 2019 \\ Articulo aprobado: 25 de Diciembre del 2019 \\ Licenciado en Ciencias de la Educación especialización Administración y Supervisión Educativa egresado de la Universidad \\ de Guayaquil (Ecuador) con 8 años de experiencia en la docencia en Escuela Educación Básica Dr. Alfredo Pareja \\ Diezcanseco. Maestrante en la Universidad Nacional Mayor de San Marcos de Lima (Perú), mención Gestión de la \\ Educación.
}

\section{Resumen}

La investigación sobre la planificación estratégica es fundamental en el desenvolvimiento de las instituciones ya que se relaciona con la calidad del servicio, en el ámbito educativo, se analiza su relación con la calidad educativa. Por eso, la presente investigación cuantitativa, de diseño correlacional se ha propuesto determinar la relación entre la planificación estratégica y la calidad de la educación en las instituciones educativas de Nivel Básico Elemental de la Parroquia el Batan, en el periodo 2018. Para cumplir con este propósito, se ha trabajado con una muestra de 40 docentes y 12 trabajadores administrativos. Los resultados fueron descritos por dimensiones. La hipótesis fue falseada con la prueba estadística ChiCuadrado. Se concluyó que existe relación significativa entre la planificación estratégica y la calidad de la educación entre de los docentes y personal administrativo de las instituciones educativas. Además, se dedujo con la prueba de correlación $($ Rho de Spearman $=816$ y Sig. $=$ $0,000)$ que existe una correlación positiva fuerte entre las variables de estudio.

Palabras claves: Planificación estratégica, calidad educativa, servicio educativo.

\section{Abstract}

The present research entitled Teaching performance with the educational quality, of the educational unit "Republic of Ecuador" which aims to determine the relationship between the variables of teaching performance and educational quality. The research is of a basic type, of correlational descriptive scope, the design used is non-experimental, of a crosssectional nature. The sample was of 100 teachers, of the educational unit "Republic of Ecuador". To collect the data, the survey was used as a technique for both variables, using the questionnaire as an instrument, which was applied to the sample after going through a validity and reliability process. Data processing was performed with the SPSS software (version 23). Performed the descriptive analysis and the correlation through Spearman's Rho coefficient, with a result of Rho $=0.878^{* *}$, interpreted as a very good correlation between the variables, with a $\rho=$ 0.00 ( $\mathrm{p}<0.05)$, with which rejects the null hypothesis, therefore, the results indicate that there is a relationship between teaching performance and educational quality in the particular educational institution of the "Republic of Ecuador" educational unit.

\section{Keywords: Teaching performance, educational quality, teachers, institution.}

\section{Sumário}

A pesquisa sobre planejamento estratégico é essencial no desenvolvimento das instituições, no que se refere à qualidade do serviço; no campo educacional, é analisada sua relação com a qualidade educacional. Portanto, a presente pesquisa quantitativa, de desenho correlacional, foi proposta para determinar a relação entre o planejamento estratégico e a 


\section{Ciencia y Educación (ISSN 2707-3378) \\ Vol. 1 No. 2 \\ Febrero del 2020}

qualidade da educação nas instituições de ensino fundamental da Paróquia de Batan, no período de 2018. Para cumprir com isso Para isso, trabalhou com uma amostra de 40 professores e 12 trabalhadores administrativos. Os resultados foram descritos por dimensões. A hipótese foi falsificada com o teste estatístico do qui-quadrado. Concluiu-se qu'existe uma relação significativa entre o planejamento estratégico e a qualidade da educação entre professores e funcionários administrativos das instituições de ensino. Além disso, deduziu-se com o teste de correlação (Rho de Spearman = 816 e Sig. $=0,000)$ qu'existe uma forte correlação positiva entre as variáveis do estudo.

Palavras-chave: Planejamento estratégico, qualidade educacional, serviço educacional.

\section{Introducción}

En el ámbito educativo, el docente es un actor fundamental ya que sobre el recae la formación de los estudiantes. La formación docente en Ecuador, según el Informe Nacional sobre el desarrollo de la Educación (2004), ha sido deficiente ya que los continuos cambios en el sistema de formación no han solucionado los problemas básicos como el divorcio del currículo con la sociedad actual.

La inexistencia de la planificación institucional, la limitada oferta ocupacional, el desmedro de la imagen de los docentes, los salarios bajos y la pérdida de reconocimiento social a su labor son las conclusiones a las que se arribaron. Ante esta problemática, se plantearon dos estrategias de acción: la reforma curricular para la educación básica y la reorientación de la formación docente.

El modelo de calidad planteado por el Ministerio de Educación de Ecuador abarca cuatro estándares: gestión educativa, Desempeño profesional, estándares de aprendizaje y estándares de infraestructura. La presente investigación se enmarca en los estándares de Gestión Educativa. Este criterio hace "referencia a procesos de gestión y prácticas institucionales que contribuyen a la formación deseada de los estudiantes. Además, favorecen el desarrollo profesional de los actores de la institución educativa y permiten que esta se aproxime a su funcionamiento ideal" (Ministerio de Ecuador, s.f., p.7).

La investigación es fundamental ya que relaciona dos conceptos fundamentes: planteamiento estratégico y calidad de la educación. La información sistematizada puede ser base para investigaciones en el ámbito educativo. La exploración temática permite desarrollar conceptos que clarifiquen el tema de la calidad y la planificación estratégica. Estos dos temas resultan vitales al momento de un desarrollo efectivo de la educación.

En ese sentido, la presente investigación se ha propuesto responder a la siguiente interrogante: ¿En qué medida la planificación estratégica se relaciona con la calidad de la educación en las Instituciones educativas de nivel básico elemental de la Parroquia El Batan, en el periodo 2018?

Por ello, se ha propuesto como objetivo general determinar la relación que existe entre la planificación estratégica y la calidad de la educación en las Instituciones educativas de nivel básico elemental de la Parroquia El Batan, en el periodo 2018 .

\section{Desarrollo}

Está temática ha sido desarrollada por varios autores. Salas (2013) se propone revisar la fundamentación teórica de la calidad en la gestión educativa, analizar las fortalezas $\mathrm{y}$ oportunidades que tiene en la actualidad, analizar el clima organizacional del cuerpo docente, identificar la satisfacción de los clientes (internos y externos) e identificar la deserción del alumnado de la unidad educativa. Para ello, utilizó encuestas, entrevistas, 


\section{Ciencia y Educación (ISSN 2707-3378) \\ Vol. 1 No. 2 \\ Febrero del 2020}

cuestionarios y fichas de entrevistas que le permitieron concluir que es necesario que el incrementar la infraestructura física de la unidad educativa, incorporar la información del entorno familiar en la planificación del proceso educativo.

En el campo educativo, la planificación estratégica es fundamental para el desenvolvimiento y la garantía de la calidad educativa. Corredor (2001, p.44) propone que la planificación estratégica debe ser considerada como un "proceso [por el] cual se provee lograr situaciones objetivas, determinando poder $\mathrm{y}$ potencialidad de actores que actúen en escenarios cambiantes con tiempos críticos y bajo condiciones de incertidumbre".

Según Venegas (2006), el origen de la planificación está relacionado a la publicación del libro de Albert Waterston (1964), en el que se plasman las lecciones de la experiencia extraída del informe solicitado por el Banco Mundial y de informes realizados sobre los países industrializados. Los puntos básicos para comprender el contexto de la planificación en el ámbito de la educación son los siguientes:

- La planificación surgió en los países del tercer mundo como respuesta a demandas de gobiernos y organismos para racionalizar la cooperación financiera, y no como producto de una conciencia propia.

- La planificación fue básicamente macroeconómica y hacia grandes inversiones en infraestructura, y nunca contempló los concomitantes requerimientos del desarrollo social de cada país.

- La planificación estuvo divorciada de los procesos y estructuras políticas, de suerte que, una vez que los planes servían para apoyar los créditos externos, los políticos simplemente daban la espalda a los planificadores y a sus planes.

(Venegas 2006, pp. 8-9)

El contexto del origen de la planificación expuesto por Venegas (2006) muestra que la necesidad de la planificación en el ámbito educativo se genera por la desidia de la clase política de los países latinoamericanos. Incluso, se advierte que la cooperación financiera no es producto de las búsquedas de las demandas internas sino de imposiciones de organismos extranjeros. La Planificación estratégica, según Manes (2004), es viable si se considera elementos básicos como evaluación del entorno, visión, misión, metas y objetivos, estrategias y acciones, entre otros. Los elementos básicos de la planificación estratégica son seis:

\section{a. Evaluación del entorno}

La evaluación del entorno se realiza a través de FODA, la cual evalúa las oportunidades y amenazas del afuera por medio de indicadores o fuentes secundarias de información, incluso también las primarias por medio de investigaciones ad hoc realizadas en la comunidad amplia. Las fortalezas y debilidades del adentro investigando las expectativas y percepciones de la comunidad educativa. (Manes, 2005, p. 20)

\section{b. Visión}

Según Manes (2004), la visión es la "percepción posible, deseable, realista y creíble del futuro de la institución educativa" (p. 22). Por ello, debe ser conocida por toda la institución ya que es fuente de inspiración y motivación en la comunidad educativa.

\section{c. Misión}

Según Manes (2004, p. 22), la misión de una institución educativa se visibiliza en los "principios fundamentales, en el ideario, en el lema, en el himno y en todos aquellos elementos 
que la distingan y le otorguen identidad propia”. La misión debe responder a las necesidades de la comunidad educativa.

\section{d. Metas y objetivos}

Las metas y los objetivos dentro de una institución son propósitos cuantificables, los cuales están planificados y se les asigna responsables.

\section{e. Estrategias y acciones}

Según Manes (2004) la estrategia es un "método de pensamiento para clasificar y jerarquizar los acontecimientos, con la finalidad de diseñar e implementar procesos institucionales más efectivos" (p.22).

f. Organización y sistemas de control Para establecer la planificación estratégica, es fundamental desarrollar una planeación de actividades.

La planificación estratégica se estructura en forma de cascada, donde cada integrante desempeña el rol que le corresponde según su estructura de poder. Según Serna (2003), la planificación estratégica se presenta en tres niveles que interactúan entre ellos: Planeación estratégica corporativa, planeación estratégica funcional y planeación estratégica operativa.

El término "calidad" es polisémico y controversial; sin embargo, fundamental. Es polisémico ya que está presente en diversas áreas del conocimiento. En el plano educativo, por ejemplo, para Braslavsky (2006) "una educación de calidad es aquella que permite que todos aprendan lo que necesitan aprender, en el momento oportuno de su vida y de sus sociedades y en felicidad". Así la calidad está relacionada con el concepto de bienestar y desarrollo del ser humano.
Braslavsky (2006) plantea que diez factores para una educación de calidad para todos en el siglo XXI:

1. El foco en la pertinencia personal y social

La educación deber ser pertinente, eficaz y eficiente.

2. La convicción, la estima y la autoestima de los involucrados

El segundo factor de calidad de la educación es una tensión creativa entre la convicción, la estima y la autoestima de las sociedades y de las dirigencias políticas y de las administraciones en el valor de la educación

3. La fortaleza ética y profesional de los maestros y profesores

4. La capacidad de conducción de los directores e inspectores

5. El trabajo en equipo dentro de la escuela y de los sistemas educativos

6. Las alianzas entre las escuelas y los otros agentes educativos

7. El currículo en todos sus niveles

8. La cantidad, calidad y disponibilidad de materiales educativos

9. La pluralidad y calidad de las didácticas

10. Los mínimos materiales y los incentivos socioeconómicos y culturales

UNESCO, por otro lado, considera que la Calidad de la Educación está relacionada con la igualdad. Bajo este parámetro, UNESCO planteó dos abordajes:

a. La calidad de la educación en función de sistemas y en términos de insumos (inputs), procesos y productos (outputs).

b. La calidad de la educación en el marco sectorial se establece en tres factores primordiales: relevancia, efectividad $\mathrm{y}$ eficiencia. 


\section{Ciencia y Educación (ISSN 2707-3378) \\ Vol. 1 No. 2 \\ Febrero del 2020}

\section{- Relevancia:}

Reconoce que los objetivos establecidos y los contenidos prescritos para la educación son contenciosos y a menudo tienen que ser negociados, es decir, deben estar relacionados a las necesidades de la comunidad.

\section{- Efectividad:}

La efectividad se refiere a la medida en qué los objetivos y las metas establecidas están siendo alcanzadas

\section{- Eficiencia}

La eficiencia se refiere a la búsqueda qué hace falta para que se cumplan las promesas de educación de calidad. Se trata de analizar si pudiesen lograrse mejores resultados con los mismos recursos o si los mismos resultados podrían ser alcanzados con menos recursos. En la Ley Orgánica de Educación Intercultural de Ecuador, se plantearon cinco estándares. Estándares de Aprendizaje que describen los logros y constituyen referentes comunes que los estudiantes deben alcanzar a lo largo de la trayectoria escolar: desde el primer grado de la Educación General Básica hasta el tercer curso de Bachillerato Estándares de Gestión Escolar (Ministerio de Educación, s.f.p.19).

- Estándares de Desempeño

\section{Estándares de Desempeño Profesional Directivo}

Es referente al liderazgo, a la gestión pedagógica, al talento humano, a recursos, al clima organizacional y a la convivencia escolar; para asegurar su influencia efectiva en el logro de aprendizajes de calidad de todos los estudiantes en las instituciones educativas a su cargo (Ministerio de Educación, s.f.p.15).
2. Dimensiones del desempeño profesional directivo

Los estándares de Desempeño Profesional Directivo hacen referencia al liderazgo, a la gestión pedagógica, al talento humano, a recursos, al clima organizacional y a la convivencia escolar para asegurar su influencia en el logro de aprendizajes de los estudiantes.

\section{Estándares de Desempeño Profesional Docentes}

El propósito de los estándares de Desempeño Profesional Docente es fomentar en el aula una enseñanza que permita que todos los estudiantes ecuatorianos alcancen los perfiles de egreso o aprendizajes declarados por el Currículo Nacional para la Educación General Básica y para el Bachillerato (Ministerio de Educación, s.f.p.11).

4. Estándares de Infraestructura Escolar

Son criterios normativos para la construcción y distribución de los espacios escolares, que buscan satisfacer requerimientos pedagógicos y aportar al mejoramiento de la calidad en la educación. Estos estándares enuncian las condiciones de infraestructura que deben cumplir progresivamente todas las instituciones educativas con la finalidad de alcanzar niveles óptimos de calidad en el proceso de enseñanzaaprendizaje (Ministerio de Educación, s.f.p.47).

\section{Estándares de Gestión Escolar}

Hacen referencia a procesos de gestión y prácticas institucionales que contribuyen a la formación deseada de los estudiantes. Además, favorecen el desarrollo profesional de los actores de la institución educativa y permiten que esta se aproxime a su funcionamiento ideal (Ministerio de Educación, s.f.p.7). 


\section{Ciencia y Educación (ISSN 2707-3378) \\ Vol. 1 No. 2 \\ Febrero del 2020}

\author{
6. Las dimensiones de la Gestión \\ escolar
}

Las dimensiones de la Gestión escolar favorecen el desarrollo profesional de todos los actores educativos. Además, vigilan el cumplimiento de los lineamientos y las disposiciones establecidos por el Ministerio de Educación.

\section{Metodología}

\section{Identificación de variables}

1. Variable: Planificación estratégica

A. Por su naturaleza: Atributiva

B. Por la posesión de las características: Continuas

C. Por el método de la medición de las variables: Cuantitativa

D. Por el número de valores que adquieren las variables: Politomías

2. Variable: Calidad de la educación

A. Por su naturaleza: Atributiva

B. Por la posesión de las características: Continuas

C. Por el método de la medición de las variables: Cuantitativa

D. Por el número de valores que adquieren las variables: Politomías

\section{Presentación de resultados}

En la presente investigación se procedió a medir las variables en su relación con los docentes y el personal administrativo de las instituciones educativas de nivel básico elemental de la Parroquia El Batan, en el periodo 2018. En ese sentido, en la relación de planificación estratégica y la calidad educativa se obtuvo como resultado el coeficiente de correlación de Rho de Spearman que es igual a 0,816 por lo que se determinó que existe una correlación positiva fuerte entre las variables de estudio. Por tanto, se puede afirmar que es viable mejorar la planificación estratégica para mejorar la calidad educativa en las instituciones educativas. Este resultado concuerda con las investigaciones internacionales realizadas por Ramos Vergara (2012), Salas (2013) y Flores (2013).

Para Ramos (2012), el equipo directivo ejerce sobre los miembros de la institución un fuerte liderazgo y la tarea no se ve truncada en el proceso. Así mismo, la formación docente de la Institución revela un nivel calificado para el desempeño de las funciones; pues todos tienen estudios de tercer nivel; lo que constituye una garantía en la condición académica del personal que se dedica a la tarea educativa; más aún, ahora, que la nueva ley de educación exige este requerimiento.

En la investigación de Salas (2013) se concluyó que el incrementar la infraestructura física de la unidad educativa, incorporar la información del entorno familiar en la planificación del proceso educativo, se obtuvo mejores resultados en la calidad educativa. Por otra parte, para Flores (2013) que la institución cuenta con todos los documentos administrativos para realizar una excelente gestión educativa; sin embargo, estos documentos al parecer no han sido socializados entre todos los miembros de la comunidad educativa especialmente entre los docentes y estudiantes.

A nivel nacional, la investigación concuerda con Castro (2017), Ramírez (2015) y Romero (2017). Para Castro (2017), el coeficiente de rho de Spearman $=0,897$ indica correlación alta y sig. biliateral $=0,000$ que es menor 0.05 , es decir, existe una relación significativa entre la planificación estratégica del directivo y calidad del servicio educativo de la institución educativa N 3014 "Leoncio Prado" del distrito del Rímac - 2017. Mientras que para Ramírez (2015), existe una relación directa y 


\section{Ciencia y Educación (ISSN 2707-3378) \\ Vol. 1 No. 2 \\ Febrero del 2020}

significativa entre el planeamiento estratégico y la calidad de gestión de acuerdo con el coeficiente de Spearman. Finalmente, para Romero (2017), la planificación estratégica influye significativamente con la calidad de la gestión educativa de la Universidad Técnica de Babahoyo. Al aplicarse el estadístico de Pearson se encontró una correlación de 0.782 , lo cual indica una correlación positiva considerable

\section{Conclusión}

El objetivo principal de este artículo fue determinar la relación que existe entre la planificación estratégica y la calidad de la educación en las Instituciones educativas de nivel básico elemental de la Parroquia El Batan, en el periodo 2018.

\section{Planificación estratégica y calidad de la educación}

Se observó que el $50 \%$ de personas encuestadas entre docentes y personal administrativo opinan que la calidad de la educación es regular y que la planificación estratégica está medianamente completa. Además, el $25 \%$ de personas opinan que la calidad de la educación es casi siempre buena y la planificación estratégica está completa. Según la prueba de independencia $($ Chi cuadrado $=52,00$ y Sig. $=0,000)$, se concluye que existe relación significativa entre la planificación estratégica y la calidad de la educación de los docentes y personal administrativo de las instituciones educativas. Se dedujo, según prueba de correlación (Rho de Spearman $=816$ y Sig. $=0,000)$ que existe una correlación positiva fuerte entre las variables de estudio.

La planificación estratégica y la calidad de educación en el área de relevancia
Se observó que $50 \%$ de personas encuestadas entre docentes y personal administrativo opinan que la calidad de la educación en el área de relevancia es regular y que la planificación estratégica está medianamente completa. Además, 25\% personas opinan que la calidad de la educación en el área de relevancia es casi siempre buena y además la planificación estratégica está completa. Según la prueba de independencia (Chi cuadrado $=52,00$ y Sig. $=$ $0,000)$, se concluye que existe relación significativa entre la planificación estratégica y la calidad de la educación en el área de relevancia entre de los docentes y personal administrativo de las instituciones educativas de nivel básico elemental de la Parroquia El Batan, en el periodo 2018 .

La planificación estratégica y la calidad de educación en el área de eficiencia y eficacia

Se observó que $50 \%$ personas encuestadas entre docentes y personal administrativo opinan que la calidad de la educación en el área de eficiencia y eficacia es regular y que la planificación estratégica está medianamente completa. El 25\% de personas opinan que la calidad de la educación en el área de eficiencia y eficacia es casi nunca buena. Además, el 25\% afirmó que la planificación estratégica está medianamente completa. Según la prueba de independencia (Chi cuadrado $=5,778$ y Sig. $=$ 0,016), se concluye que existe relación significativa entre la planificación estratégica y la calidad de la educación en el área de eficiencia y eficacia en los docentes y el personal administrativo de las instituciones educativas de nivel básico elemental de la Parroquia El Batan, en el periodo 2018.

La planificación estratégica y la calidad de educación en el área de equidad 


\section{Ciencia y Educación (ISSN 2707-3378) \\ Vol. 1 No. 2 \\ Febrero del 2020}

Se observó que el $50 \%$ personas encuestadas entre docentes y personal administrativo opinan que la calidad de la educación en el área de equidad es regular y que la planificación estratégica está medianamente completa. Además, el 25\% personas opinan que la calidad de la educación en el área de equidad es casi nunca buena y además la planificación estratégica está medianamente completa. Según la prueba de independencia (Chi cuadrado $=$ 5,778 y Sig. $=0,016$ ), se concluye que existe relación significativa entre la planificación estratégica y la calidad de la educación en el área de equidad en los docentes y el personal administrativo de las instituciones educativas de nivel básico elemental de la Parroquia El Batan, en el periodo 2018 .

A las instituciones educativas, se les sugiere elaborar el PEI con la participación de los docentes, agentes educativos, y el personal administrativo que conoce la funcionalidad de las oficinas. Para garantizar la eficiencia y la eficacia, se sugiere al director hacer uso de los recursos institucionales y establecer fechas de ceremonias para realizar reconocimiento a los miembros de la institución de forma pública. Así mismo, sería recomendable establecer concursos considerando las inteligencias múltiples y las diversas disciplinas de estudio. A los directores de las instituciones educativas se les sugiere establecer un liderazgo educativo para lograr la inclusión, el acceso y la permanencia de la comunidad educativa.

\section{Referencias bibliográficas}

Alarcón Mujica (2013) Gestión educativa y calidad de la educación en instituciones privadas en Lima metropolitana. [Tesis de Maestría] Instituto para la Calidad de la Educación, Universidad de San Martín de Porres, Lima. Recuperado de http://www.repositorioacademico.usmp.edu. pe/bitstream/usmp/1168/1/alarcon_msz.pdf
Alarcón M. (2008). Planificación estratégica: una herramienta en la gestión escolar a nivel de educación básica. [Tesis de Maestría]. Universidad Nacional experimental de Guayana, Guyana. Recuperado de http://cidar.uneg.edu.ve/DB/bcuneg/EDOC S/TESIS/TESIS_POSTGRADO/MAESTRI AS/CIENCIAS_EDUCACION/TGMLA53 T462008.pdf

Bracho Pérez, Kleeder y Carruyo Duran, Norcelly (2011) Planificación estratégica y gestión del gerente educativo en instituciones de educación primaria. Revista Praxis, 7, pp.13-29. Recuperado de DialnetPlanificacionEstrategicaYGestionDelGerent eEducativ-5907174.pdf

Castro Santamaria, Mariella (2017) Planificación estratégica del directivo y calidad del servicio educativo en la IE 3014 Leoncio Prado del distrito del Rímac 2017 [Tesis de Maestría] Facultad de Educación, Universidad César Vallejo, Lima. Recuperado de http://repositorio.ucv.edu.pe/bitstream/handl e/UCV/9950/Castro_SM.pdf?sequence=1\&i sAllowed $=\mathrm{y}$

Corredor, J. (2001). La planificación Estratégica. Caracas - Venezuela. Vadell Hermanos. Editores, C. A

Chipana Cahuapaza, Máximo (2015) Gestión pedagógica y la calidad educativa en las Unidades de Gestión Educativa local de San Román y Azángaro-2013 [Tesis de doctorado]. Universidad Andina, Juliaca. Recuperado de http://repositorio.uancv.edu.pe/bitstream/ha ndle/UANCV/276/TESIS.pdf?sequence $=1$ \&isAllowed $=\mathrm{y}$

Flores, José (2013) Incidencia de la Planificación Estratégica en la gestión de la calidad de los ámbitos de la gerencia educativa. [Tesis de Maestría] Universidad Anina Simón Bolívar, Ecuador. Recuperado del

http://repositorio.uasb.edu.ec/bitstream/106 44/3320/1/T1238-MGE-FloresIncidencia.pdf

Ibarra Alva, Américo (2012) Planeamiento estratégico y su relación con la gestión de la calidad de la Institución Educativa 


\section{Ciencia y Educación (ISSN 2707-3378) \\ Vol. 1 No. 2 \\ Febrero del 2020}

Kumamoto I N³092- Puente Piedra - Lima - año 2011 [Tesis de Maestría]. Facultad de Educación, Universidad Nacional Mayor de San Marcos, Lima. Recuperado de http://cybertesis.unmsm.edu.pe/bitstream/ha ndle/cybertesis/1674/Ibarra_aa.pdf?sequenc $\mathrm{e}=1$

Hernández R, Fernández C y Baptista P (2006). Metodología de la Investigación. Cuarta Edición. Editorial Mc Graw Hill. México

Manes, Manuel (2004) Gestión estratégica para instituciones educativas. 2 da edición. Buenos Aires, Granica.

Mario, G. (2005). Planeamiento Estratégico: El caso del centro de extensión universitaria y proyección social (CEUPS) de la Facultad de Ciencias Administrativas de la UNMSM (2005-2009) [Tesis de Maestría] Lima: Universidad Nacional Mayor de San Marcos.

OEI (2004) Informe Nacional sobre el desarrollo de la educación. $47^{\circ}$ Reunión de la conferencia Internacional de educación, CIE. Recuperado de https://www.oei.es/historico/quipu/ecuador/ ibeecuador.pdf

ONU (2010). Datos mundiales de educación. VII Ed. 2010/2011. Recuperado de http://www.ibe.unesco.org/fileadmin/user_u pload/Publications/WDE/2010/pdf-

versions/Ecuador.pdf

PREAL, Fundación Ecuador, Contrato Social por la Educación y Grupo FARO (2006). La calidad con equidad: El desafío de la educación ecuatoriana. Informe de Progreso Educativo. ECUADOR. Recuperado de http://B3n/preal_ecuador2006.pdf

Ramírez Maldonado, Yenncy (2015) El planeamiento estratégico y su relación con la calidad de gestión en las instituciones educativas públicas del nivel primario de Chorrillos-Lima, 2013. [Tesis de doctorado] Instituto para la Calidad de la Educación, Universidad San Martín de Porres, Lima. Recuperado de http://www.repositorioacademico.usmp.edu. pe/bitstream/usmp/1962/1/ramirez_myp.pdf

Ramos Vergara, Gustavo (2012) Planificación estratégica en el colegio Internacional Rudolf Steiner de Quito [Tesis de Maestría]
Universidad Virtual Escuela de Graduados en Educación. Toluca, México. Recuperado de

https://repositorio.itesm.mx/bitstream/handl e/11285/571902/DocsTec_12846.pdf?seque nce $=1 \&$ isAllowed $=\mathrm{y}$

Romero Robles, P. (2016). La planificación estratégica y su influencia en la calidad de gestión educativa de la Universidad Técnica de Babahoyo. Journal of Science and Research: Revista Ciencia E Investigación, 1 (2),

20-22. https://doi.org/https://doi.org/10.26910/issn. 2528-8083volliss2.2016pp20-22

Sainz de vicuña Ancín, J. (2009). El plan estratégico en la práctica. (2da ed.). Madrid: ESIC Editorial.

Salas C. (2005). Planificación estratégica para mejorar la gestión gerencial del gerente educativo en educación media diversificada y profesional. [Tesis de maestría no publicada]. Universidad Rafael Belloso Chacín

Salas Bustos y Lucín Arboleda (2013) Evaluación de la calidad del servicio educativo para determinar el nivel de la deserción estudiantil en la Unidad Educativa "Capitán Pedro Osacr Salas Bajaña" [Tesis de Maestría] Universidad Católica de Santiago de Guayaquil, Ecuador. Recuperado de http://repositorio.ucsg.edu.ec/bitstream/331 7/2421/1/T-UCSG-POS-MAE-45.pdf

Serna, H. (2003). Gerencia Estratégica. 6ta Edición. Santa Fe de Bogotá - Colombia. 3R Editores.

Venegas Jiménez, Pedro (2006) Planificación educativa: bases metodológicas para su desarrollo en el siglo XXI, 2da edición, San José: EUNED

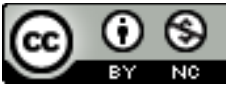

Creative Commons Reconocimiento-No Comercial 4.0 Internacional. Copyright (c) Marcos Oswaldo García Romero 